\title{
Computed assisted surgery for replacement of the temporomandibular joint with customized prostheses. Can we validate the results?
}

\author{
Luis Alejandro Boccalatte ${ }^{1,2,3}$ (D) M. G. Nassif ${ }^{1}$ - M. F. Figari ${ }^{1,3} \cdot$ N. L. Gómez ${ }^{1} \cdot$ L. E. Ritacco ${ }^{4}$
}

Received: 3 December 2020 / Accepted: 7 December 2020 / Published online: 5 January 2021

(C) Springer-Verlag GmbH Germany, part of Springer Nature 2021

We would like to start thanking Dr. Sembronio and collaborators for their careful review of our work and insightful comments. Although they have considered our research on replacement of the temporomandibular joint interesting, they have questioned several aspects of our study, which we would like to respond in detail below.

First, although in the introduction of our paper we stated that we found no validation reports, published, we were specifically referring to scientific papers validating the triad of planning, navigation, and custom-made implants. In the Discussion we quoted, among others, the study by Sembronio et al., which does not validate navigation but the use of titanium cutting guides instead. Also, our validation system did not compare positioning of initial and final prosthetic components (condyle and glenoid), but established that difference in absolute values according to the position of the heads of the screws used in each prosthetic component. For that reason, we stated that there were no published studies similar to ours.

Luis Alejandro Boccalatte

luis.boccalatte@hospitalitaliano.org.ar;

1 Head and Neck - Craniomaxillofacial Surgery Section, General Surgery Department, Hospital Italiano de Buenos Aires, Juan D. Perón 4190, C1181ACH Buenos Aires, Argentina

2 Academic Department of Morphological Sciences, Instituto Universitario, Hospital Italiano de Buenos Aires, Buenos Aires, Argentina

3 Academic Department of Surgery, Instituto Universitario, Hospital Italiano de Buenos Aires, Buenos Aires, Argentina

4 Computer Assisted Surgery (CAS Unit), Argentina - Consejo Nacional de Investigaciones Científicas y Técnicas (CONICET), Instituto de Medicina Traslacional e Ingeniería Biomédica (IMTIB), Hospital Italiano de Buenos Aires, Buenos Aires, Argentina
Secondly, the fusion between preoperative planning and the final result was performed using algorithms of manual and automated recording of the preoperative and postoperative computed tomograms. Two fusions were performed for each case: one fusion of the maxilla for the glenoid and one fusion of the mandibular ramus to assess the condyle. We agree with Sembronio et al. when they state: "This plays a crucial role in determining the precision of measurement and this is especially true in those cases where occlusal changes are planned by repositioning the maxilla and the mandible."

Thirdly, we agree that navigation entails certain limitations, as do other reported techniques. However, final clinical results of our patients' occlusion have been very satisfactory. We acknowledge that titanium cutting guides offer very good results, as shown in several studies, but we prefer to use navigation for two reasons: one is that we lack experience with the use of guides for this procedure, and the other is that in our country the cost of navigation is lower.

Finally, it seems that the authors of the letter have misinterpreted the objective of our study. Our objective was to establish an adequate validation system, and to be able to audit our own results. We did not mean to suggest that this system should be used universally that was certainly far from our intention. Although in absolute number discrepancies were found between planning and postoperative results, we reiterate that clinical results of the final occlusion (as shown in the clinical photos of the paper) were very satisfactory.

We are grateful for the opportunity of discussing these important issues, since we believe that the exchange of experience and ideas among investigators is always fruitful and productive.

Publisher's note Springer Nature remains neutral with regard to jurisdictional claims in published maps and institutional affiliations. 